\title{
Cloning, Sequencing and Phylogenetic Analysis of inv E Gene of Salmonella typhimurium
}

\author{
Yashpal Singh ${ }^{1}$, Apoorv Tiwari ${ }^{1}$, Rajesh Kumar ${ }^{2}$ and M.K. Saxena ${ }^{3 *}$ \\ ${ }^{1}$ Department of Molecular Biology and Genetic Engineering, ${ }^{2}$ Department of Veterinary \\ Microbiology, ${ }^{3}$ Department of Veterinary Physiology \& Biochemistry, G.B. Pant University of \\ Agriculture \& Technology, Pantnagar, Uttarakhand, India \\ *Corresponding author
}

\begin{tabular}{|l|}
\hline K e y w o r d s \\
Salmonella, Gene, \\
Protein, Vaccine
\end{tabular}

\section{Introduction}

Salmonellosis is one of the most common food-borne diseases of humans and animals. In human beings there are two forms of
Salmonellosis. These are Typhoidal and Nontyphoidal Salmonellosis (Gal-Mor et al., 2014). Like other gram-negative bacteria virulence of Salmonella is regulated by chromosomal and plasmid mediated genes 
(Singh et al., 2018). Multiple drug resistance have been found to be associated with the infections of Salmonella (Tamuly et al., 2014; Kalaiyarasu et al., 2008; Verma et al., 2014) which produced difficulties in treatment of disease.

Though by the use of modern biological tools efforts have been made to differentiate organism at molecular level by analysis of plasmid profile (Shivshankara et al., 2000), DNA markers (Verma et al., 2014; Saxena et al., 2012) and protein based markers (Singh et al., 2018).

The studies exhibited high genetic variations among the field isolates. Therefore, there is a need of targeting conserved proteins for development of safe and effective vaccine against Salmonella. Efforts have been made by targeting outer membrane proteins (Saxena et al., 2012; Pandey et al., 2015; Jha et al., 2015) along with new generation adjuvant system (Tamuly and Saxena, 2012; Tamuly et al., 2014) and a protection upto a certain level could be achieved in lab animal models. In the continuation of same Inv $E$ protein can be targeted for development of suitable vaccine against Salmonella. Inv $\mathrm{E}$ is a virulence factor and responsible for invasion of organism in host cell (Shi et al., 2012).

So it can be considered as toxin factor of Salmonella which may be proven as an efficient target for vaccine development. But to be proven as vaccine candidate inv E should fulfill few pre-requisites. Firstly, it should be a conserved protein among the various serovars and secondly, it must have effective B cell and T cell epitopes.

Therefore, in present study we cloned and sequenced inv E gene. The conserved domain and phylogenetic analysis of inv $\mathrm{E}$ was made to deduce the conservation status of the protein among the various serovars.

\section{Materials and Methods}

\section{Bacterial strain}

The field isolate of Salmonella typhimurium (A-201) used in this study was obtained from Animal Biotechnology Center, G.B. Pant University of Agriculture \& Technology, Pantnagar. The culture was revived and the purity of the culture was determined by Salmonella specific PCR and biochemical test specific to Salmonella. Further, culture was maintained in Hektoen enteric agar slants and glycerol stocks during the study. E. coli DH5á used in cloning was purchased from Bangalore GeNei, India and grown in Luria Bertani broth while blunt cloning vector pJET 1.2, blunting enzyme, and T4 DNA ligase were procured from Qiagen, USA. Ampicillin $(100 \mu \mathrm{g} / \mathrm{ml})$ used for selection of recombinants were procured from Himedia, India.

\section{Cloning of inv E gene}

Genomic DNA of Salmonella typhimurium was isolated by CTAB method. The sequence information available on NCBI as well as gene tool software was used for designing primers for inv E gene of Salmonella typhimurium.

inv E (Forward): 5' GGA TCC ATG ATT CCT GGT TCC ACC TCC 3'

\section{inv E (Reverse): 5' AAG GTT TTA AGA CGG CTT TTC AAT AGT ACG A 3'}

Amplification of inv E gene was carried out with $50 \mu \mathrm{l}$ of PCR reaction mixture containing $20 \mathrm{ng}$ of genomic DNA (template), $200 \mu \mathrm{M}$ dNTPs, 20 picomoles of each primer, 5 ul of 10X AccuTaq ${ }^{\mathrm{TM}}$ LA PCR buffer with 3 unit of Jumpstart $^{\mathrm{TM}}$ AccuTaq $^{\mathrm{TM}}$ LA (Sigma). Final volume of PCR reaction mixture was making up to $50 \mu \mathrm{l}$ by using sterilized water. The gene was amplified by under following cycling conditions, initial denaturation at $94^{\circ} \mathrm{C}$, 
followed by denaturation at $94^{\circ} \mathrm{C}$, annealing at $54^{\circ} \mathrm{C}$, and elongation at $68^{\circ} \mathrm{C}$. PCR product was analyzed in $1.5 \%$ agarose gel and the size of the amplicon was measured by comparing with standard molecular weight marker. The elution of PCR product was carried out from agarose gel using Qiagen mini elute gel extraction kit. Further, PCR product was cloned in pJET vector by blunt end cloning. After blunting of PCR product, $1 \mu \mathrm{l}$ of pJET vector and $1 \mu \mathrm{l}$ of T4 DNA ligase were added and ligation was carried out at $22^{\circ} \mathrm{C}$ for 4 hours. The ligated product ( $5 \mathrm{ul}$ ) was checked on $1.5 \%$ agarose gel and transformed into E.coli DH5a cells by Calcium chloride method (Sambrook et al., 1989). Recombinants were screened by selection on the basis of antibiotic resistance and analyzed for the presence of insert by colony PCR. The insert was released by double digestion with Hind III and Bam $\mathrm{H} 1$ and the size of insert was measured by comparing with standard molecular weight marker. Plasmid DNA was isolated from clones and the selected clones were sent to University of Delhi, South Campus for sequencing. The deduced sequences were submitted to NCBI.

\section{Open reading frame}

The Open reading frame (ORF) of inv E gene of Salmonella typhimurium was deduced by using Gene tool software.

\section{GC content}

The percentage of GC content in inv E gene of Salmonella typhimurium was calculated by using DNA/RNA GC content calculator. (http://www.endmemo.com/bio/gc.php).

\section{Conserved domain search}

Conserved domain analysis of the inv $\mathrm{E}$ protein sequence was performed by using $\mathrm{CD}$ Search tool of NCBI.
Sequence similarity and phylogenetic analysis

The deduced sequence was analyzed for homology using BLASTn (http://www.ncbi. nlm.nih.gov/). The sequences showing maximum similarity with inv $\mathrm{E}$ gene were subjected to multiple sequence alignment and a phylogenetic tree was constructed based on the comparative analysis of related sequences using MEGA (Molecular Evolution Genetics Analysis) tool at nucleotide level.

The analysis was performed on the default values of the MEGA software and Neighbourjoining statistical method at 1000 bootstrap replication was used for tree construction.

\section{Results and Discussion}

The purity of the procured Salmonella typhimurium culture was confirmed using biochemical characterization and PCR with Salmonella typhimurium specific primers. The culture was found to be MR+, VP- and Urease- biochemically, which represents characteristic of Salmonella typhimurium.

\section{PCR Amplification and Cloning}

The PCR amplification of inv $\mathrm{E}$ gene of Salmonella typhimurium was conducted with isolated genomic DNA and gene specific primers resulting in a product of approximate size 1119 bp (Fig 1). The PCR product was purified using QIAGEN quick gel extraction kit, cloned in pJET 1.2 cloning vector (Fermentas, USA) and transformed into E.coli DH5á cells. Further, recombinant clones were selected by colony PCR (Fig. 2). Plasmid with inv $\mathrm{E}$ gene insert was isolated and sequenced at University of Delhi, South Campus. The sequence with complete cds of inv $\mathrm{E}$ gene was submitted in NCBI Gene Bank (Accession number KC607873). 


\section{Open reading frame analysis}

The sequence showed a complete ORF of 1119 bp using Gene tool software. (Fig 3)

\section{GC content analysis}

GC content varies with different genes. The length of the coding sequence is directly proportional to higher $\mathrm{G}+\mathrm{C}$ content as the stop codon has a bias towards $\mathrm{A}$ and $\mathrm{T}$ nucleotides. Therefore, shorter the sequence the higher will be the AT bias. The total GC content in the inv E gene sequence of Salmonella typhimurium was found to be $45.5 \%$.

\section{Conserved Domain Search}

Nucleotide sequence (1119 bp) was used to predict the domain region in the sequence. A single domain, type III secretion system regulator inv $\mathrm{E}$ was identified in the inv $\mathrm{E}$ gene sequence of Salmonella typhimurium. HrpJ-like domain family represents a conserved region approximately 200 residues long within a number of bacterial hypersensitivity response secretion protein HrpJ and similar proteins. HrpJ forms part of a type III secretion system through which, in phytopathogenic bacterial species, virulence factors are thought to be delivered to plant cells. This family also includes the inv $\mathrm{E}$ invasion protein from Salmonella. This protein is involved in host parasite interactions and mutations in the InvE gene render Salmonella typhimurium non-invasive. Inv $\mathrm{E} S$. typhimurium mutants fail to elicit a rapid $\mathrm{Ca}^{2+}$ increase in cultured cells, an important event in the infection procedure and internalisation of S. typhimurium into epithelial cells. This family includes bacterial SepL and SsaL proteins. SepL plays an essential role in the infection process of enterohemorrhagic Escherichia coli and is thought to be responsible for the secretion of EspA, EspD and EspB while SsaL of Salmonella typhimurium is thought to be a component of the type III secretion system.

Fig.1 1.5\% Agarose gel showing 1.1 kb PCR product of inv E gene. Fig.2 The cloned gene was confirmed by release of insert by double digestion with Bam H I and Hind III, M: $1 \mathrm{~kb}$ ladder

\section{(GeneRuler ${ }^{\mathrm{TM}}$, Fermentas)}

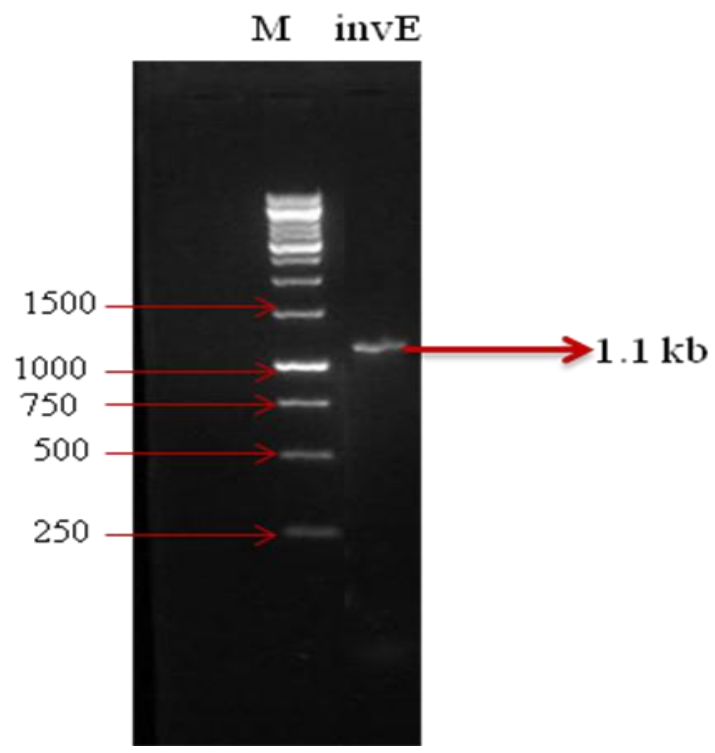

Fig.1

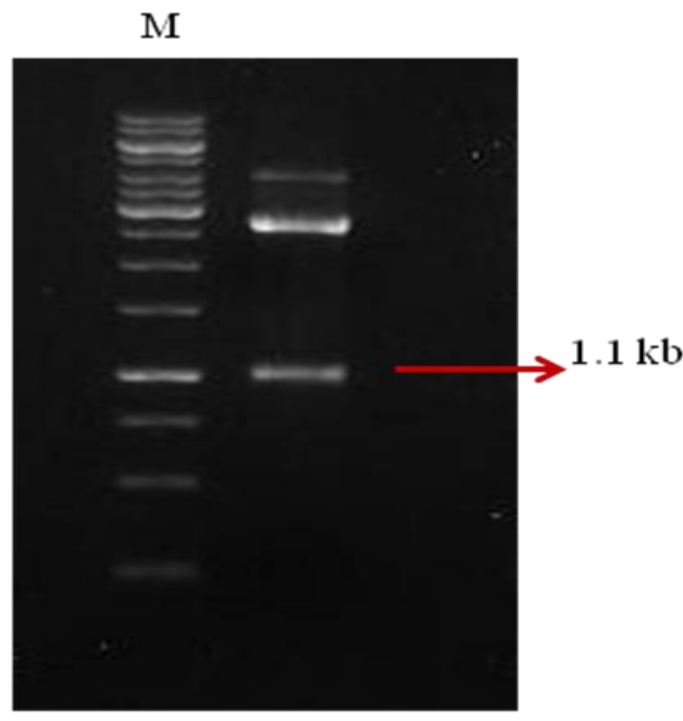

Fig.2 
Fig.3 Open reading frame (ORF) analysis of inv E gene sequence of Salmonella typhimurium (ORF 1: 1-1119)

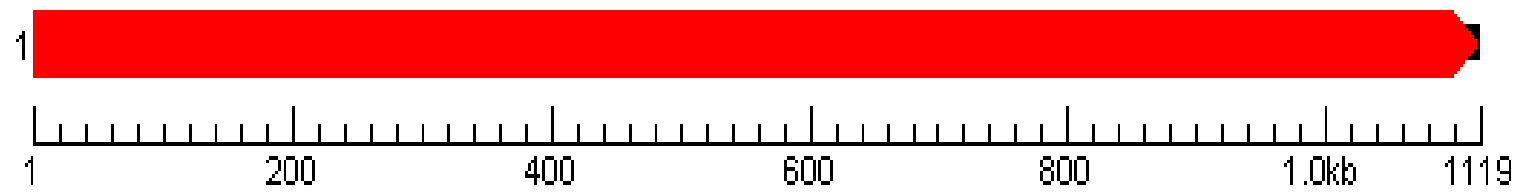

Fig.4 Total GC content in the inv E gene sequence of Salmonella typhimurium

\section{Length: 1119bp, Averge GC: 45.5\%}

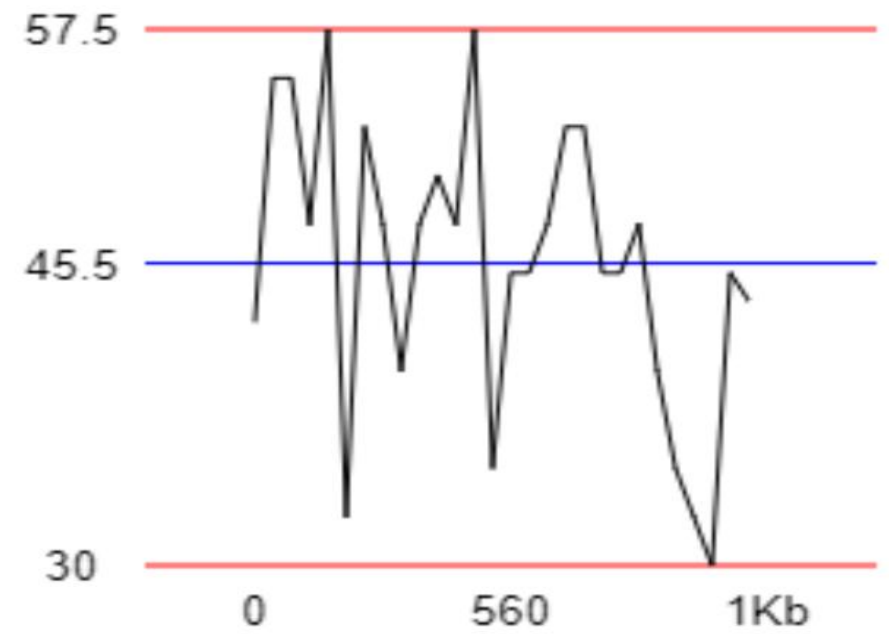

Fig.5 Conserved Domain of Inv E protein of Salmonella typhimurium

Conserved domains on [gi| 460974407|gb|AGH15701.1|]

View Concise Results $\mathbf{2}$

invasion protein [Salmonella enterica subsp. enterica serovar Typhimurium]

\section{Protein Classification}

PRK15338 family protein (domain architecture ID 11487744)

PRK15338 family protein

Graphical summary $\square$ Zoom to residue level

show extra options »

Query seq. Specific hits Superfanilies

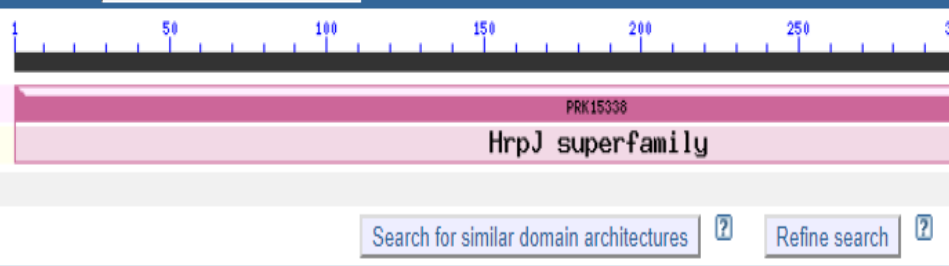

List of domain hits

+ Name Accession

[H] PRK15338 PRK15338 type II secretion system regulator InvE; Provisional
Interval E-value

1-372 0e+00 
Fig.6 Dendrogram for phylogenetic analysis at nucleotide level of inv E gene marked with red square box with other closely related sequences

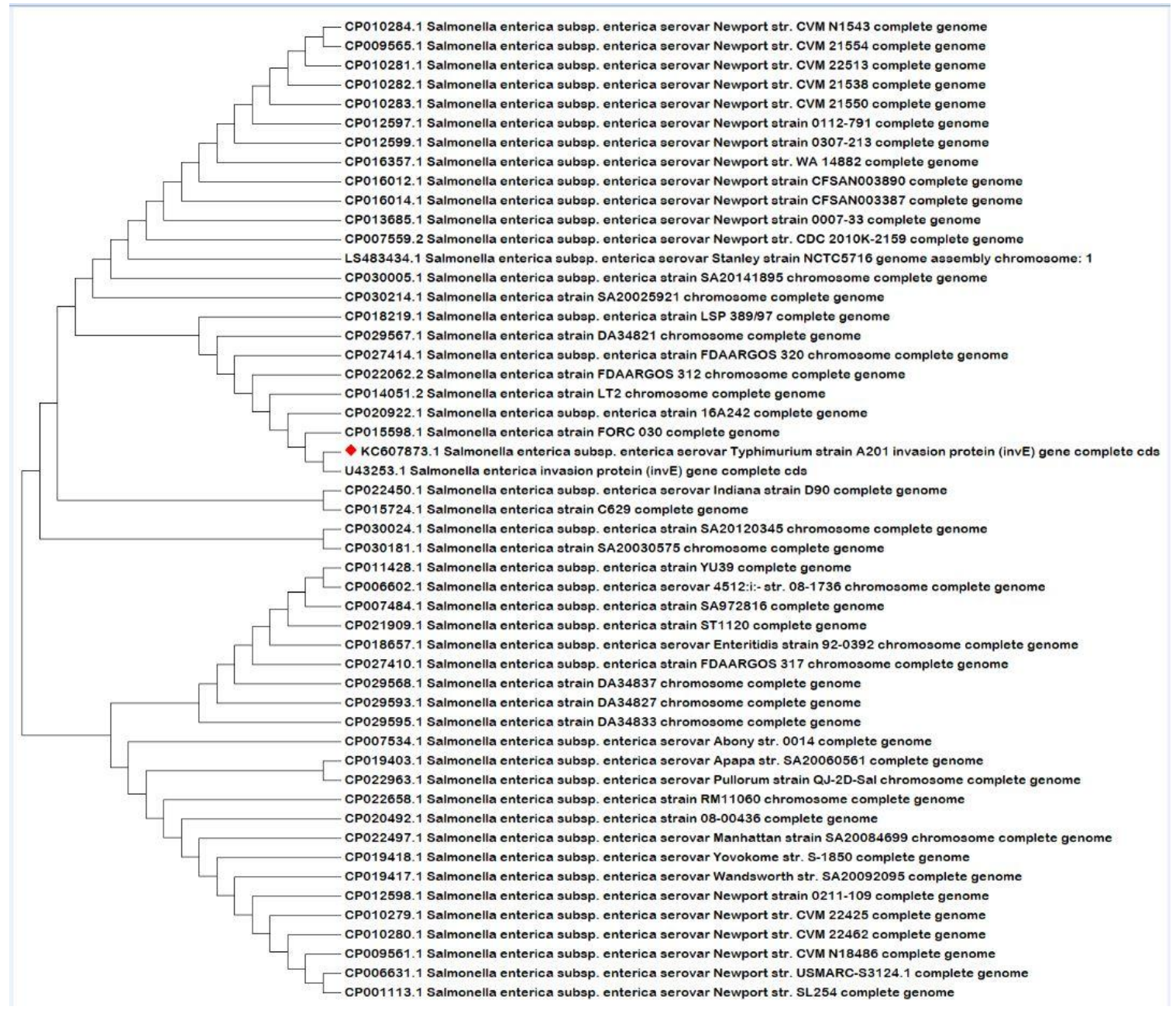

Phylogenetic analysis of inv $\mathrm{E}$ gene of $S$. typhimurium at nucleotide level

The NCBI BLASTn search of the inv E gene showed maximum homology (99\%) with Salmonella enterica subsp. enterica strain LSP 389/97 complete genome and Salmonella enterica strain DA34833 chromosome complete genome at nucleotide level (Fig. 6). Though the sequence was shared by other serovar of Salmonella like S. enteritidis.

Salmonella is an important contagious gramnegative pathogen which affects both humans and poultry. It is mainly causative agent of typhoid fever caused by $S$. typhi in humans and non-typhoidal salmonellosis caused by S.typhimurium. Both of the forms of disease result in high mortality as well as severe economic loss (Kemal, 2014). In past few years the emergence of multi-drug resistant strains of Salmonella in many countries of the world has become a major concern (Chandane et al., 2017). Vaccination is considered as the prominent option but effective vaccines are not available as currently available vaccines have certain limitations such as it cannot be used in pregnant women, debatable immune response in children and high cost of production (Ochiai et al., 2014). Although to 
overcome these problems several workers have made efforts to develop vaccine targeting recombinant as well as immunogenic outer membrane proteins of Salmonella (Bhat and Jain, 2010; Jha et al., 2015). Strategies involving new adjuvant system have also been developed (Tamuly and Saxena 2012, Tamuly et al., 2014) which produces better immune response. The invasion protein Inv $\mathrm{E}$ is an important virulence factor located on SPI-1 which is responsible for spread of Salmonella in host cells. In order to explore the relationship between pathogenicity of Salmonella and virulence factor genes (inv E), inv E gene of Salmonella typhimurium was isolated, amplified, sequenced and homology was searched for similarity with related sequences of different strains. Sequence was further used for bioinformatics analysis of Inv E protein, which exhibited a single domain. On phylogenetic analysis, sequence exhibited 99\% similarity with Salmonella enterica strain FORC030 complete genome and Salmonella enterica subsp. enterica serovar Indiana strain D90, complete genome. In our study we could observe that Inv $\mathrm{E}$ is a conserved protein in genus Salmonella as it has exhibited homology with different serovars of Salmonella like $S$. indiana, $S$. stanley, S. newport, $S$. enteritidis etc.

Simultaneously it has exhibited a complete functional conserved domain. Therefore, it fulfills the first pre-requisite as a candidate for vaccine development. In the further studies the epitopes, secondary and tertiary structure of the protein is to be deduced to explore the possibility of development of subunit vaccine.

\section{Acknowledgment}

The authors highly acknowledge the Department of Biotechnology, Govt of India for providing funds for the study. The authors are grateful to Dean, College of Veterinary and Animal Sciences and Director of Experiment Station, G. B. Pant University of Agriculture \& Technology for providing necessary facilities for this study.

\section{References}

Bhat, N.H. and Jain, S.K., 2010. Immunogenic evaluation of a recombinant 49-kilodalton outer membrane protein of Salmonella typhi as a candidate for a subunit vaccine against typhoid. Journal of Infectious Disease and Immunity, 2(2): 30-40.

Chandane, P., Gandhi, A. and Bowalekar, S. 2017. Study of antibiotic susceptibility pattern of Salmonella typhi in children suffering from enteric fever. Annals of Tropical Medicine and Public Health, 10(2): 440-443.

Gal-Mor, O., Boyle, E.C. and. Grassl, G.A. 2014. Same species, different diseases: how and why typhoidal and nontyphoidal Salmonella enterica serovars differ. Frontiers in Microbiology, 5: doi: 10.3389/fmicb.2014.00391.

Jha, R., Kumar, A., Saxena, A., Pandey, M., Kumar, R. and Saxena, M.K. 2015. Heterogeneous expression and functional evaluation of in silico characterized recombinant OmpC of Salmonella typhimurium as a functional poultry vaccine to eradicate zoonotic transmission. African Journal of Biotechnology, 14(41): 2862-2870.

Kalaiyarasu, S., Saxena, M.K., Gupta, R.S. 2013. Emerging of multiple drug resistance in Salmonella typhimurium serovars isolated from Indian Ganga River. Advances in Animal and Veterinary sciences, 1:1-3.

Kemal, J. 2014. A Review on the Public Health Importance of Bovine Salmonellosis. Journal of Veterinary Science \& Technology, 5: 2: doi: 10.4172/2157-7579.1000175. 
Ochiai R.L., Khan M.I., Soofi S.B., Sur. D., Kanungo, S., You, Y.A., Habib, M.A., Sahito, S.M., Manna, B., Dutta, S., Acosta, C.J., Ali, M., Bhattacharya SK., Bhutta, Z.A. and John, D.C.2014. Immune Response to $\mathrm{Vi}$ Capsular Polysaccharide typhoid vaccine in Children 2 to 16 years old in Karachi, Pakistan and Kolkata, India. Clin and Vaccine Immunol, 5:661-666.

Pandey, M. and Saxena, M.K. 2015. Cloning and immunopotential analysis of Omp 28 of Salmonella typhimurium for the development of subunit vaccine for poultry salmonellosis. Indian Journal of Poultry Science, 50(2):138-142.

Sambrook, J., Fritsch, E.F. and Maniatis, T. 1989. Molecular Cloning. A Laboratory Manual 198(9) edition 2nd Cold Spring Harbour Laboratory Press Cold Spring Harbour NY.

Saxena, A., Tamuly, S. and Saxena, M.K. 2012. Cloning, sequencing, and in silico characterization of Omp 28 of Salmonella typhi (strain MTCC 733) to develop r-DNA vaccine for typhoid fever. Journal of Natural Science Biological Medicine, 3(2): 133-138.

Shi, Q., Zhang, Y., Wang, Q.Y., Gao, G., Fang, H., Miao, F., Yuan, Z. and He, H. 2012. Phylogenetic analysis of virulence factor gene of Salmonella isolated from clinically symptomatic Chickens. African Journal of Microbiology Research, 6(8): 17181722.

Shivshankara, N., Singh, V.P., Saxena, M.K. and Kumar, A.A. 2000. Plasmid profile analysis of Pasteurella multocida.
Indian journal of Comparative Microbiology, Immunology and Infectious diseases, 21(2): 115-7.

Singh, Y., Saxena, A., Kumar, R. and Saxena, M.K. 2018. Isolation and Protein Profiling of Outer Membrane Proteins (Omps) of Salmonella Typhi. International Journal of Current Microbiology and Applied Sciences, 7(8): 2851-2855.

Singh, Y., Saxena, A., Kumar, R. and Saxena, M.K. 2018. Virulence System of Salmonella with Special reference to Salmonella enterica http://dx.doi.org/ 10.5772/intechopen.77210.

Tamuly, S. and Saxena, M.K. 2012. Preparation of Calcium phosphate nanoparticles and evaluation of their effects on muscle cells of rat. Current Science, 102(4): 610-612.

Tamuly, S., Saxena, M.K., Saxena, A., Mishra, R.K. and Jha, R. 2014. Comparative Evaluation of Humoral Immune Response Generated by Calcium Phosphate Nanoparticle Adjuvanted and Saponin-Adjuvanted Recombinant Outermembrane Protein 87 (Omp87) of Pasteurella multocida (Serotype B: 2) in Mice. Journal of Nanopharmaceutics Drug Delivery, 2(1): doi:10.1166/jnd.2014.1045.

Verma, J.K., Pilkhwal, D., Tamuly, S., Kumar, R., Kumar, A. and Saxena, M.K. 2014. Molecular characterization of Ampicillin resistant poultry isolates of Salmonella typhimurium. Journal of Cell and Tissue Research, 14:40194026.

\section{How to cite this article:}

Yashpal Singh, Apoorv Tiwari, Rajesh Kumar and Saxena, M.K. 2018. Cloning, Sequencing and Phylogenetic Analysis of inv E Gene of Salmonella typhimurium. Int.J.Curr.Microbiol.App.Sci. 7(11): 1700-1707. doi: https://doi.org/10.20546/ijcmas.2018.711.194 